\title{
Correction to: Perturbation effects for some nonlinear parabolic equations with lower order term and $L^{1}$-data
}

\section{Abdellaoui ${ }^{1}$ (1)}

Published online: 17 October 2020

(c) Orthogonal Publisher and Springer Nature Switzerland AG 2020

\section{Correction to: Journal of Elliptic and Parabolic Equations https://doi.org/10.1007/s41808-020-00071-z}

The author would like to correct some typo errors in the original paper [Abdellaoui, M.: Perturbation effects for some nonlinear parabolic equations with lower order term and L $^{1}$-data. J Elliptic Parabol Equ (2020). https://doi.org/10.1007/s4180 8-020-00071-z]. The considerations presented below should be inserted instead of the existing ones. This corrigendum corrects also an unfortunate mathematical and typographical error that have been forgotten in the above mentioned paper. We sincerely apologize for our mistakes.

(i) In the abstract, the $p$-Laplace operator should be $\Delta_{p} u:=\operatorname{div}\left(|\nabla u|^{p-2} \nabla u\right)$.

(ii) The boundary condition $u(t, x)=0$ on $(0, T) \times \Omega$ in problem $(\mathcal{P})$ should contain the correct form (with the symbol $\partial$ )

$$
u(t, x)=0 \quad \text { on }(0, T) \times \partial \Omega,
$$

The author wish to make the some correction to Eqs. (2.12) and (2.13), and with the modification $u_{n}(t, x)=0$ on $(0, T) \times \partial \Omega$ for (2.1).

The changes do not affect the scientific results (the manuscript will be updated and the correction will remain online on the article webpage).

Publisher's Note Springer Nature remains neutral with regard to jurisdictional claims in published maps and institutional affiliations.

The original article can be found online at https://doi.org/10.1007/s41808-020-00071-z.

M. Abdellaoui

mohammed.abdellaoui3@usmba.ac.ma

1 Laboratory of Mathematical Analysis and Applications, Department of Mathematics, Faculty of Sciences Dhar El Mahraz, Sidi Mohamed Ben Abdellah University, B.P. 1796, Atlas Fez, Morocco 